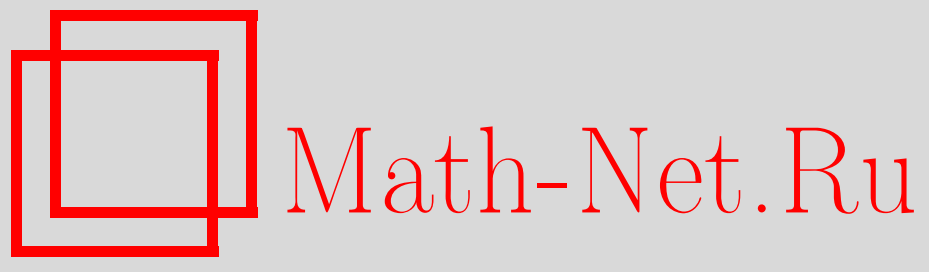

В. Ю. Протасов, Полугруппы неотрицательных матриц, УМН, 2010, том 65, выпуск 6, 191-192

DOI: https://doi.org/10.4213/rm9383

Использование Общероссийского математического портала Math-Net.Ru подразумевает, что вы прочитали и согласны с пользовательским соглашением http://www.mathnet.ru/rus/agreement

Параметры загрузки:

IP: 54.147 .182 .235

26 апреля 2023 г., 09:28:50

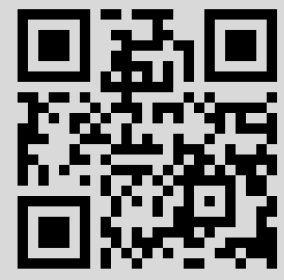




\section{Полугруппы неотрицательных матриц}

\section{В. Ю. Протасов}

Пусть $\mathscr{S}$ - произвольная полугруппа (по умножению) $(n \times n)$-матриц, все входящие в нее матрицы неотрицательны (имеют неотрицательные элементы). В частности, $\mathscr{S}$ может быть задана как множество произведений данного набора матриц. При каких условиях $\mathscr{S}$ будет содержать положительную матрицу? Данная задача возникает при изучении неоднородных цепей Маркова, уточняющих алгоритмов, показателя Ляпунова и т. д. [1]-[5]. В частном случае, когда $\mathscr{S}$ порождена одной матрицей, т. е. $\mathscr{S}=\left\{A^{k}, k \in \mathbb{N}\right\}$, известны несколько критериев, как комбинаторных, так и аналитических, а также результаты о минимальной положительной степени матрицы и о структуре матриц, не имеющих положительных степеней [6]-[9]. В данной работе мы получим критерий существования положительного элемента для произвольной полугруппы матриц (предложение 1) и докажем полиномиальную разрешимость этой задачи для полугрупп, порожденных конечным набором матриц (теорема 1). Далее сформулируем две открытые проблемы. Мы предполагаем, что выполнены условия:

(а) $\mathscr{S}$ неприводима, т. е. не существует подмножества базисных векторов, линейная оболочка которого есть общее инвариантное подпространство всех матриц из $\mathscr{S}$;

(б) у матриц полугруппы $\mathscr{S}$ нет нулевых столбцов и нулевых строк.

Если $\mathscr{S}$ порождена набором матриц, то она удовлетворяет условиям (а), (б) в точности тогда, когда им удовлетворяет порождающий набор. Везде далее $(\cdot, \cdot)$ - скалярное произведение, $\|\cdot\|_{1}-L_{1}$-норма в $\mathbb{R}^{n}$ и соответствующая операторная норма матрицы, $e_{i}$ - базисные векторы, span - линейная оболочка.

ПредЛожение 1. Полугруппа $\mathscr{S}$ при условиях (а), (б) не содержит положительной матрицы тогда и толъко тогда, когда найдется такая пара индексов $i, j$, $i \neq j$, что для любого $A \in \mathscr{S}$ имеем $a_{k i} a_{k j}=0$ при всех $k=1, \ldots, n$ (m.е. носители $i$-го и $j$-го столбцов не пересекаются).

\section{Доказательство состоит из трех лемм.}

Лемма 1. Если полугруппа $\mathscr{S}$ при условиях (а), (б), содержит матричу, имеющую положительную строку, то она содержит положительную матрицу.

ДоказАтельство. Пусть матрица $A \in \mathscr{S}$ имеет максимальное число $k \geqslant 1$ положительных строк. Считаем, что это - первые $k$ строк. Пусть $k<n ;(k+1)$-я строка содержит положительный элемент $a_{k+1, i}$. В силу (а) существует матрица $C \in \mathscr{S}$, у которой $c_{i 1}>0$. Тогда $i$-я строка матрицы $C A$ положительна. Согласно (б), для любого $B \in \mathscr{S}$ матрица $A B$ также имеет первые $k$ положительных строк. Следовательно, первые $k+1$ строк матрицы $A C A$ положительны. Таким образом, $k=n$.

В силу (б) достаточно доказать предложение 1 для стохастических (по столбцам) матриц. Положим $V=\left\{x \in \mathbb{R}^{n} \mid x_{1}+\cdots+x_{n}=0\right\}$. Ясно, что $A V \subset V$ и $\|A\|_{1}=1$ для стохастической матрицы $A$. Пусть $\mu(A)$ - кратность собственного значения 1 . Следующая лемма общеизвестна.

Лемма 2. Если $A$ - стохастическая матрица и $\mu(A)=1$, то при достаточно больших $k$ матрица $A^{k}$ имеет положительную строку.

Лемма 3. Если $\mathscr{S}$ состоит из стохастических матрии, и $\mu(A) \geqslant 2$ для всех $A \in \mathscr{S}$, то найдутся такие $i \neq j$, что $\left(A e_{i}, A e_{j}\right)=0$ для всех $A \in \mathscr{S}$.

ДокАЗАтЕльСтво от противного: пусть для любых $i \neq j$ найдется матрица $A_{i j} \in \mathscr{S}$, для которой $\left(A e_{i}, A e_{j}\right)>0$. Тогда $\left\|A_{i j} e_{i}-A_{i j} e_{j}\right\|_{1}<\left\|e_{i}-e_{j}\right\|_{1}=2$.

Работа поддержана грантами РФФИ-08-01-00208, МД-2195.2008.1 и НШ-3233.2008.1. 
Докажем, что существует $P \in \mathscr{S}$ такая, что $\left\|P e_{i j}\right\|_{1}<1$ для всех пар $(i, j)$, где $e_{i j}=\left(e_{i}-e_{j}\right) / 2$. Полагаем $P_{1}=A_{12}, I_{1}=\{(1,2)\}$ и применяем индукцию: если существует матрица $P_{k} \in \mathscr{S}$, для которой $\left\|P_{k} e_{i j}\right\|_{1}<1$ при всех $(i, j) \in I_{k}$, где $I_{k}-$ некоторое множество из $k$ пар индексов, то берем произвольную пару $(a, b) \notin I$. Пусть $a$-й и $b$-й столбцы матрицы $P_{k}$ имеют положительные координаты в строках $q$ и $r$ соответственно, $q \neq r$. Так как $\left\|P_{k}\right\|_{1}=\left\|A_{q r}\right\|_{1}=1$, то для матрицы $P_{k+1}=A_{q r} P_{k}$ имеем $\left\|P_{k+1} e_{i j}\right\|_{1}<1$ при всех $(i, j) \in I_{k+1}=I_{k} \cup\{(a, b)\}$. Итак, мы получили матрицу $P$, для которой $\left\|P_{\mid V}\right\|_{1}<1$, а значит $\mu(P)=1$. Что противоречит предположению, поскольку $P \in \mathscr{S}$.

Совмещая леммы $1-3$, получаем доказательство предложения 1.

Теорема 1. Наличие положительной матрицы в полугруппе, порожденной $m$ матрицами, удовлетворяющими (а) и (б), определяется за полиномиальное время.

ДокАзАтЕльство. Пусть $\mathscr{S}$ порождена матрицами $A_{1}, \ldots, A_{m}$. Рассмотрим направленный граф, вершины которого - упорядоченные пары $\left(i_{1}, i_{2}\right), 1 \leqslant i_{1}, i_{2} \leqslant n$. Ребро, ведущее из вершины $\left(j_{1}, j_{2}\right)$ в вершину $\left(i_{1}, i_{2}\right)$, существует тогда и только тогда, когда найдется $k=1, \ldots, m$, для которого оба элемента $a_{i_{1}, j_{1}}$ и $a_{i_{2}, j_{2}}$ матрицы $A_{k}$ положительны. Согласно предложению $1, \mathscr{S}$ содержит положительную матрицу тогда и только тогда, когда из любой вершины графа можно дойти до диагонали, т. е. до одной из вершин вида $(i, i)$. Определяя последовательно вершины первого уровня, второго и т. д., мы проверяем данное условие. Алгоритм затрачивает $m n^{3}(1+o(1))$ операций, а объем входных данных равен $m n^{2}$.

Проблема 1. Пусть $N(m)$ - минимальное число такое, что если полугруппа $\mathscr{S}$, заданная набором из $m$ матрии, имеет положительный элемент, то он является произведением не более $N$ матрии, данного набора. Какова оченка сверху на $N(m)$ ?

Для полугрупп, порожденных одной матрицей, известно несколько оценок [6]-[8].

ПроБлема 2. Верно ли, что полугруппа $\mathscr{S}$, удовлетворяющая (а), (б), не содержит положительной матрицы тогда и только тогда, когда существует разбиение $\{1, \ldots, n\}=\bigcup_{k=1}^{r} U_{k}, r \geqslant 2$, такое, что каждая матрица $A \in \mathscr{S}$ задает перестановку множеств $U_{1}, \ldots, U_{r}$, m.е. существует перестановка $\sigma=\sigma_{A}$ множества $\{1, \ldots, r\}$, для которой $U_{k} \subset \mathscr{U}_{\sigma(k)}$ при всех $k$, где $\mathscr{U}_{k}$ есть $\operatorname{span}\left\{e_{i}, i \in U_{k}\right\}$.

Последнее условие очевидным образом достаточно для отсутствия положительной матрицы в $\mathscr{S}$. Вопрос: будет ли оно необходимым? Для полугрупп, порожденных одной матрицей, это известный результат [5; гл. 8], равносильный теореме Романовского [9]. Заметим, что без условий (а) и (б) утверждение неверно.

\section{Список литературы}

[1] Р. Л. Добрушин, Матем. сб., 34:3 (1954), 541-556. [2] Y. Wang, J. Approx. Theory, 113:2 (2001), 207-220. [3] E. S. Key, J. Theoret. Probab., 3:3 (1990), 477-488. [4] R. M. Jungers, V. Protasov, V. D. Blondel, Linear Algebra Appl., 428:10 (2008), 2296-2311. [5] В. Ю. Протасов, Функи. анализ и его прил., 44:3 (2010), 84-88. [6] Р. Хорн, Ч. Джонсон, Матричный анализ, Наука, М., 1989, 656 с. [7] Ф. Р. Гантмахер, Теория матрии, Наука, М., 1966, 576 с. [8] M. Akelbek, S. Kirkland, Linear Algebra Appl., 430:4 (2009), 1111-1130. [9] V. Romanovsky, Bull. Soc. Math. France, 61 (1933), 213-219.

\section{В. Ю. Протасов (V. Yu. Protasov)}

Московский государственный университет им. М. В. Ломоносова

E-mail: v-protassov@yandex.ru
Представлено В. М. Тихомировым Принято редколлегией 24.06.2010 